\section{Decompositions for control systems on manifolds with an} affine connection

\author{
Andrew D. Lewis* $\quad$ Richard M. Murray ${ }^{\dagger}$
}

06/04/1996

Last updated: $22 / 08 / 1998$

\section{Abstract}

In this letter we present a decomposition for control systems whose drift vector field is the geodesic spray associated with an affine connection. With the geometric insight attained with this decomposition, we are able to easily prove some special results for this class of control systems. Examples illustrate the theory.

Keywords. affine connection, symmetric product, controllability, mechanics

AMS Subject Classifications. 53B05, 53B20, 53C22, 70E15, 70H33, 70Q05, 93B03, 93B05, $93 \mathrm{~B} 29$

\section{Introduction}

In a recent paper, Lewis and Murray [1997] introduce the notion of "configuration controllability" for a class of mechanical control systems and present some results for this notion of controllability. In the statement of their results, the so-called symmetric product provides a valuable shorthand. For Levi-Civita connections, the symmetric product was originally seen in the work of Crouch [1981] on gradient control systems. The geometric meaning of the symmetric product on manifolds with a general affine connection is given ] In this letter we combine the results of Lewis and Murray [1997] with the interpretation of the symmetric product by Lewis [1998] to obtain a decomposition of control systems whose drift vector field is the geodesic spray associated with a general affine connection. Examples of such control systems include Lagrangian mechanical systems whose Lagrangian is the kinetic energy with respect to a Riemannian metric. However, the use of general affine connections is more than simply an easily performed abstraction. Synge constrained equations of motion for a mechanical in terms of an affine connection which is in general not a Levi-Civita connection. Other authors have done similar work along these lines since the work of Synge. In the control *Assistant Professor, Department of Mathematics and Statistics, Queen's University, Kingston, ON K7L 3N6, CANADA

Email: andrewomast.queensu.ca, URL: http://www. mast. queensu.ca/“andrew/

Research in part supported by NSF Grant CMS-9502224

Worked performed while a Research Fellow in Control and Dynamical Systems at the California Institute

of Technology.

Associate Professor, Control and Dynamical Systems, Mail Code 104-44 California Institute of Technology, Pasadena, CA 91125, U.S.A.

Email: murray@indra.caltech.edu, URL: http://avalon.caltech.edu/〜murray/ context, we mention a recent paper of Bloch and Crouch [1995]. This puts the associated constrained control systems in the framework of the present paper and so provides impetus to study control systems associated with general affine connections. The decompositions we obtain are entirely analogous to the decompositions one obtains for nonlinear control systems which reduce to the Kalman controllability decomposition for linear systems.

In Section 2 we review the necessary concepts from the theory of affine connections and also review the results of [Lewis 1998]. In Section 3 we present the control systems we will consider. These systems have a phase space which is the tangent bundle of a manifold. It is common to be interested only in the control of the points on the manifold and not be concerned with their velocities. Apropos to this, we review the controllability definitions of [Lewis and Murray 1997]. With the results on the symmetric product presented in Section 2, we are able to concisely restate the main controllability results of [Lewis and Murray 1997]. When the system is not controllable (by our definition), we are able to succinctly describe the reachable sets in terms of geometry of the affine connection. In Section 4 we use our main results of Section 3 to arrive at some natural corollaries. Thus the contribution of the paper is threefold. First, we make clear the geometry of the results of [Lewis and Murray 1997]; second, we describe a natural decomposition of the systems we are considering in cases when they are not controllable; third, we present some new results which are easily derived from the general theory. Examples which illustrate our results are given in Section 5 .

\section{Affine connections}

We refer the reader to [Kobayashi and Nomizu 1963] for a discussion of affine connections. Let $M$ be an $n$-dimensional manifold. We denote by $C^{\infty}(M)$ the set of $C^{\infty}$ functions on $M$ and by $\mathscr{T}(M)$ the set of $C^{\infty}$ vector fields on $M$. An affine connection on $M$ assigns to every pair of vector fields $X$ and $Y$ on $M$ a vector field $\nabla_{X} Y$ with the assignment satisfying

AC1. the map $(X, Y) \mapsto \nabla_{X} Y$ is $\mathbb{R}$-bilinear,

AC2. $\nabla_{f X} Y=f \nabla_{X} Y$ for $f \in C^{\infty}(M)$ and $X, Y \in \mathscr{T}(M)$, and

AC3. $\nabla_{X}(f Y)=f \nabla_{X} Y+\left(\mathscr{L}_{X} f\right) Y$ for $f \in C^{\infty}(M)$ and $X, Y \in \mathscr{T}(M)$.

If we define $\nabla_{X} f=\mathscr{L}_{X} f$ for $f \in C^{\infty}(M)$ and $X \in \mathscr{T}(M)$, then we may extend $\nabla_{X}$ to a derivation of the tensor algebra of $M$ in a unique manner. The vector field $\nabla_{X} Y$ is called the covariant derivative of $Y$ along $X$. If $c:[a, b] \rightarrow M$ is a piecewise smooth curve, then $Y$ is parallel along $c$ if $\nabla_{\dot{c}(t)} Y(c(t))=0$ for each $t \in[a, b]$. If $c$ is a curve such that its tangent vector field is parallel along the curve itself (i.e., $\left.\nabla_{\dot{c}(t)} \dot{c}(t)=0\right)$ then $c$ is a geodesic of $\nabla$. The torsion of $\nabla$ is the $(1,2)$ tensor field $T$ defined by $T(X, Y)=\nabla_{X} Y-\nabla_{Y} X-[X, Y]$ for $X, Y \in \mathscr{T}(M)$. If $T=0$ then we say $\nabla$ is torsion-free.

Let $g$ be a Riemannian metric on $M$. There exists a unique affine connection $\stackrel{g}{\nabla}$ on $M$ with the properties

LC1. $\stackrel{g}{\nabla}$ is torsion-free, and

LC2. $\stackrel{g}{\nabla}_{X} g=0$ for $X \in \mathscr{T}(M)$. 
This affine connection is called the Levi-Civita connection associated with $g$. The geodesics of the Levi-Civita connection have the property that they locally determine the paths of shortest length between two points.

2.1 RemarK: The link between geodesics of a Levi-Civita connection and Lagrangian mechanics is as follows. Let $g$ be a Riemannian metric on $M$ and define a Lagrangian function on $T M$ by $L(v)=\frac{1}{2} g(v, v)$ (i.e., the kinetic energy with respect to the Riemannian metric). Then it is easy to show that a curve $c:[a, b] \rightarrow M$ is a solution of Lagrange's equations with the Lagrangian $L$ if and only if $c$ is a geodesic for the Levi-Civita connection equations with the Lagrangian $L$ if and only if $c$ is a geodesic for the Levi-Civita connection
associated with $g$.

In a set of coordinates $\left(x^{1}, \ldots, x^{n}\right)$ for $M$, the Christoffel symbols, $\Gamma_{i j}^{k}$, for a general affine connection $\nabla$ are defined by

$$
\nabla_{\frac{\partial}{\partial x^{i}}} \frac{\partial}{\partial x^{j}}=\Gamma_{i j}^{k} \frac{\partial}{\partial k}
$$

One may verify that an affine connection is torsion-free if and only if $\Gamma_{i j}^{k}=\Gamma_{j i}^{k}, i, j, k=$ $1, \ldots, n$. The equations for a geodesic then have the form

$$
\ddot{x}^{i}+\Gamma_{j k}^{i} \dot{x}^{j} \dot{x}^{k}=0, \quad i=1, \ldots, n .
$$

These second-order equations are equivalent to the system of first-order equations on $T M$ given by

$$
\begin{aligned}
& \dot{x}^{i}=v^{i}, \quad i=1, \ldots, n \\
& \dot{v}^{j}=-\Gamma_{k l}^{j} l^{k} v^{l}, \quad j=1, \ldots, n
\end{aligned}
$$

which defines a vector field on $T M$ called the geodesic spray of $\nabla$ which we denote by $Z_{g}$.

Now we review the concepts of [Lewis 1998]. Let $D$ be a distribution on a manifold $M$ with an affine connection $\nabla$. Classically, a submanifold $N$ of $M$ is said to be totally geodesic if every geodesic whose initial velocity is tangent to $N$ remains on $N$. We wish to weaken this notion. We say a distribution $D$ is geodesically invariant if for every geodesic $c:[a, b] \rightarrow M$ of $\nabla, \dot{c}(a) \in D_{c(a)}$ implies that $\dot{c}(t) \in D_{c(t)}$ for each $t \in[a, b]$. We say that an integrable distribution $D$ is geodesically generated if it is the involutive closure of a geodesically invariant distribution.

We make a few remarks which will be useful in our discussion of control theory in Section 3.

2.2 Remarks: 1. There are distributions which are geodesically invariant but which are not integrable.

2. If $L(D)$ is the involutive closure of a gendesically invariant distribution $D$, i.e., if $L(D)$ is geodesically generated by $D$, then obviously those geodesics whose initial configuration lies in a maximal integral manifold $\Lambda$ of $L(D)$, and whose initial velocity lies in $D$, will evolve so as to remain on $\Lambda$.

The symmetric product between $X, Y \in \mathscr{T}(M)$ is the vector field

$$
\langle X: Y\rangle=\nabla_{X} Y+\nabla_{Y} X
$$

We shall denote by $\Gamma^{\infty}(D)$ the set of sections of a distribution $D$. The main result of [Lewis $1998]$ is the following theorem.

2.3 Theorem: A distribution $D$ is geodesically invariant if and only if $\langle X: Y\rangle \in \Gamma^{\infty}(D)$ for every $X, Y \in \Gamma^{\infty}(D)$.

\section{Control systems on manifolds with an affine connection}

In this section we introduce the class of control systems we will study, we present controllability definitions, and we present decompositions for these systems. Our motivation for studying systems whose drift vector field is the geodesic spray of a general affine connection comes in part from Remark 2.1. We also remind the reader of the work of Synge [1928] mentioned in the introduction which enables one to cast mechanical systems with constraints in the general formulation we consider.

Let $\nabla$ be an affine connection on a manifold $M$ and let $Y_{1}, \ldots, Y_{m}$ be linearly independent vector fields on $M$. We shall consider control systems of the form

$$
\nabla_{\dot{c}(t)} \dot{c}(t)=u^{a}(t) Y_{a}(c(t)) .
$$

Here $a$ is summed over $1, \ldots, m$. This equation is readily seen to be equivalent to the first order system

$$
\dot{v}(t)=Z_{g}(v(t))+u^{a}(t) Y_{a}^{\mathrm{lift}}(v(t))
$$

for $v \in T M$ where $Z_{g}$ is the geodesic spray associated with $\nabla$. Here $Y_{a}^{\text {lift }}$ is the vertical lift of $Y_{a}$ which is the vector field on $T M$ defined by

$$
Y_{a}^{\mathrm{lift}}\left(v_{x}\right)=\left.\frac{\mathrm{d}}{\mathrm{d} t}\right|_{t=0}\left(v_{x}+t Y_{a}(x)\right)
$$

for $v_{x} \in T_{x} M$ (see [Abraham and Marsden 1978]). In this way we obtain a first-order nonlinear control system on $T M$ whose drift vector field is the geodesic spray $Z_{g}$.

We consider controls from the sets

$$
\mathscr{U}_{T}=\left\{u:[0, T] \rightarrow \mathbb{R}^{m} \mid u \text { is piecewise constant }\right\} .
$$

A solution of (3.1) is a pair $(c, u)$ where $c:[0, T] \rightarrow M$ is a curve on $M$ and $u \in \mathscr{U}_{T}$. We define the reachable sets as follows. Let $x_{0} \in M$ and let $U$ be a neighbourhood of $x_{0}$ in $M$. Define

$\mathcal{R}_{M}^{U}\left(x_{0}, T\right)=\{x \in M \mid$ there exists a solution $(c, u)$ of $(3.1)$ such that $\dot{c}(0)=0_{x_{0}}, c(t) \in U$ for $t \in[0, T]$, and $\left.\dot{c}(T) \in T_{x} M\right\}$

and denote

$$
\mathcal{R}_{M}^{U}\left(x_{0}, \leq T\right)=\bigcup_{0 \leq t \leq T} \mathcal{R}_{M}^{U}\left(x_{0}, t\right) .
$$

Here $0_{x} \in T_{x} M$ denotes the zero vector in the tangent space. The essential feature of these definitions is that we restrict ourselves to studying the points in $M$ (not $T M$ ) which can be reached from a state whose initial velocity is zero. The velocity of the final state is unspecified.

We may now state our versions of controllability. 
3.1 Definition: We shall say that (3.1) is locally configuration accessible at $x_{0} \in M$ if there exists $T>0$ such that $\mathcal{R}_{M}^{U}\left(x_{0}, \leq t\right)$ contains a non-empty open subset of $M$ for all neighbourhoods $U$ of $x_{0}$ and all $0<t<T$. If this holds for any $x_{0} \in M$ then the system is called locally configuration accessible.

To state the controllability results, we need some notation. Let $\mathscr{V}$ be a family of vector fields on $M$. The smallest subset of $\mathscr{T}(M)$ which contains $\mathscr{V}$ and which is closed under Lie bracket we denote by $\operatorname{Lie}(\mathscr{V})$. This is known as the involutive closure of $\mathscr{V}$. The smallest subset of $\mathscr{T}(M)$ containing $\mathscr{V}$ and which is closed under symmetric product we denote by $\overline{\operatorname{Sym}}(\mathscr{V})$ and call the symmetric closure of $\mathscr{V}$. Although the work in [Lewis and Murray 1997 is presented in the context of Levi-Civita connections, the results are equally valid for general affine connections. The verification of this is simply a check of the relevant Lie bracket formulas for the control system on $T M$. These formulas are

$$
\begin{aligned}
{\left[Z_{g}, Y_{a}^{\text {lift }}\right]\left(0_{x}\right) } & =-Y_{a}(x) \\
{\left[Y_{b}^{\text {lift }},\left[Z_{g}, Y_{a}^{\text {lift }}\right]\right] } & =\left\langle Y_{a}: Y_{b}\right\rangle^{\text {lift }}
\end{aligned}
$$$$
\left[\left[Z_{g}, Y_{a}^{\text {lift }}\right],\left[Z_{g}, Y_{b}^{\text {lift }}\right]\right]\left(0_{x}\right)=\left[Y_{a}, Y_{b}\right](x)
$$

and are easily proved with coordinate computations. Note that in the first and third of these equations, we are naturally regarding $T_{x} M \subset T_{0} T M$ as may be done on the zero section in $T M$.

Now we are ready to state one of the main results of Lewis and Murray [1997].

3.2 Theorem: Let $\mathscr{Y}=\left\{Y_{1}, \ldots, Y_{m}\right\}$. We suppose that $\overline{\operatorname{Sym}}(\mathscr{Y})$ and $\overline{\operatorname{Lie}}(\overline{\operatorname{Sym}}(\mathscr{Y}))$ define distributions on $M$ of constant rank which we denote by $C_{\mathrm{ver}}$ and $C_{\mathrm{hor}}$, respectively. Then $\mathcal{R}_{M}^{U}(x, \leq T)$ is an open subset of the maximal integral manifold of $C_{\mathrm{hor}}$ through $x$.

It may also be shown that the set of velocities accessible from $0_{x}$ is an open subset of $\left(C_{\text {ver }}\right)_{x}$

Now we recall our discussion regarding geodesically invariant and geodesically generated distributions in Section 2. From Theorem 2.3 it is clear that $C_{\text {ver }}$ is the smallest geodesically invariant distribution containing the input vector fields $\mathscr{Y}$. By Remark $2.2-2, C_{\text {hor }}$ is the distribution geodesically generated by the inputs $\mathscr{Y}$. Theorem 3.2 may also be stated in

3.3 Theorem: Let $x \in M$ and denote by $\Lambda$ the maximal integral manifold through $x$ of the distribution geodesically generated by the inputs. For each neighbourhood $U$ of $x$ and for each $T>0$ sufficiently small, $\mathcal{R}_{M}^{U}(x, \leq T) \subset \Lambda$ and $\mathcal{R}_{M}^{U}(x, \leq T)$ contains a non-empty open subset of $\Lambda$.

All the hard work in the proof of this theorem is contained in Theorems 2.3 and 3.2 Nevertheless, Theorem 3.3 is itself important since it identifies how the geometry of the problem enters into the description of the reachable configurations.

If we choose a set of coordinates $\left(x^{1}, \ldots, x^{k}, y^{1}, \ldots, y^{n-k}\right)$ such that the maximal integral manifolds of $C_{\text {hor }}$ are given by $y^{1}=\cdots=y^{n-k}=$ constant, then the system (3.1) has a coordinate representation of the form

$$
\begin{aligned}
& \ddot{x}^{\sigma}+\Gamma_{\rho}^{\sigma} \dot{x}^{\rho} \dot{x}^{\tau}+\Gamma_{\rho \beta}^{\sigma} \dot{x}^{\rho} \dot{y}^{\beta}+\Gamma_{\beta \rho}^{\sigma} \dot{y}^{\beta} \dot{x}^{\rho}+\Gamma_{\beta \gamma}^{\sigma} \dot{y}^{\beta} \dot{y}^{\gamma}=u^{a} Y_{a}^{\sigma}, \quad \sigma=1, \ldots, k \\
& \ddot{y}^{\alpha}+\Gamma_{\rho \tau}^{\alpha} \dot{x}^{\rho} \dot{x}^{\tau}+\Gamma_{\rho \beta}^{\alpha} \dot{x}^{\rho} \dot{y}^{\beta}+\Gamma_{\beta \rho}^{\alpha} \dot{y}^{\beta} \dot{x}^{\rho}+\Gamma_{\beta \gamma}^{\alpha} \dot{y}^{\beta} \dot{y}^{\gamma}=0, \quad \alpha=1, \ldots, n-k
\end{aligned}
$$

where $\Gamma_{\rho \tau}^{\alpha}(x, y) \dot{x}^{\rho} \dot{x}^{\tau}=0$ if $\dot{x} \in C_{\text {ver }}(x, y)$. Here the Christoffel symbols of the affine connection are defined by

$$
\begin{array}{ll}
\nabla_{\frac{\partial}{\partial x^{\rho}}} \frac{\partial}{\partial x^{\tau}}=\Gamma_{\rho \tau}^{\sigma} \frac{\partial}{\partial x^{\sigma}}+\Gamma_{\rho \tau}^{\alpha} \frac{\partial}{\partial y^{\alpha}}, & \nabla_{\frac{\partial}{\partial x^{\rho}}} \frac{\partial}{\partial y^{\beta}}=\Gamma_{\rho \beta}^{\sigma} \frac{\partial}{\partial x^{\sigma}}+\Gamma_{\rho \beta}^{\alpha} \frac{\partial}{\partial y^{\alpha}}, \\
\nabla_{\frac{\partial}{\partial y^{\beta}}} \frac{\partial}{\partial x^{\rho}}=\Gamma_{\beta \rho}^{\sigma} \frac{\partial}{\partial x^{\sigma}}+\Gamma_{\beta \rho}^{\alpha} \frac{\partial}{\partial y^{\alpha}}, & \nabla_{\frac{\partial}{\partial y^{\beta}}} \frac{\partial}{\partial y^{\gamma}}=\Gamma_{\beta \gamma}^{\sigma} \frac{\partial}{\partial x^{\sigma}}+\Gamma_{\beta \gamma}^{\alpha} \frac{\partial}{\partial y^{\alpha}} .
\end{array}
$$

Observe that if $\dot{y}(0)=0$ and if $\dot{x}(0) \in C_{\mathrm{ver}}(x(0), y(0))$ then $\dot{y}(t)=0$ for $t>0$. Therefore, if we start with zero initial velocities as our controllability definitions suggest, in local coordinates our system is simply governed by the equations

$$
\ddot{x}^{\sigma}+\Gamma_{\rho \tau}^{\sigma} \dot{x}^{\rho} \dot{x}^{\tau}=u^{a} Y_{a}^{\sigma}, \quad \sigma=1, \ldots, k
$$

which comprise a locally configuration accessible control system of the form (3.1) on each of the maximal integral manifolds of $C_{\mathrm{hor}}$. This is consistent with our Remark 2.2-2.

It is worth pointing out that the decomposition (3.3) provides for us the same information as the Kalman controllability decomposition provides for linear systems. In the linear case, one may simplify the system to the form

$$
\dot{x}=A_{11} x+A_{12} y+B_{1} u
$$$$
\dot{y}=A_{22} y \text {. }
$$

The reason that the term $\Gamma_{\rho \tau}^{\alpha} \dot{x}^{\rho} \dot{x}^{\tau}$ vanishes when $\dot{x} \in C_{\text {ver }}$ in the $y$-part of equation (3.3) is analogous to $A_{21}$ being zero in (3.4).

\section{An application of the main result}

We consider a situation where the system has a natural geodesically invariant distribution. Again we consider the case when $M$ comes equipped with a Riemannian metric $g$. Now we suppose that $H$ is a Lie group which acts on $M$ from the left by isometries. For $h \in H$ we denote by $\Phi_{h}$ the associated diffeomorphism of $M$ induced by the group action. For $\xi \in \mathfrak{h}$, the Lie algebra of $H$, we define the infinitesimal generator associated with $\xi$ to be the vector field on $M$ given by

$$
\xi_{M}(x)=\left.\frac{\mathrm{d}}{\mathrm{d} t}\right|_{t=0} \Phi_{\exp (t \xi)}(x) .
$$

Now define the momentum map corresponding to this group action as the map $\boldsymbol{J}: T M \rightarrow$ $\mathfrak{h}^{*}$ determined by

$$
\left\langle\boldsymbol{J}\left(v_{x}\right) ; \xi\right\rangle=g\left(\xi_{M}(x), v_{x}\right)
$$

We refer the reader to [Marsden and Ratiu 1999] for a discussion of the above concepts. There the reader will also find a proof of the fact that for $\mu \in \mathfrak{h}^{*}, \boldsymbol{J}^{-1}(\mu)$ is invariant under the geodesic flow on $T M$. This is in particular true for $\mu=0$. Furthermore, we note that since $\boldsymbol{J}$ is linear in the fibre variables on $T M, \boldsymbol{J}^{-1}(0)$ is a distribution on $M$ (assuming that 0 is a regular value of $\boldsymbol{J})$. Therefore $D \triangleq \boldsymbol{J}^{-1}(0)$ is a geodesically invariant distribution. There are many examples where $D$ is not integrable and hence not geodesically generated. 
One may readily verify that $X \in \mathscr{T}(M)$ is such that $X^{\text {lift }}$ is tangent to $D \subset T M$ if and only if $X \in \Gamma^{\infty}(D)$, the set of sections of $D$. Therefore, any actuation of the system by control vector fields in $D$ will maintain the conservation law $\boldsymbol{J}=0$. Note that this in system cannot be local phase space $T M$ from points on $\boldsymbol{J}^{-1}(0)$. Nevertheless, it is possible that the system be locally configuration accessible. This is illustrated by the robotic leg example in Section 5 .

Let us expand on this a bit further. If we use inputs which span $D$, or equivalently, use a maximal set of inputs which maintain the conservation law, then $\mathcal{R}_{M}^{U}(x, \leq T)$ will be an open subset of a maximal integral manifold of the involutive closure of $D$. In particular, if $D$ is maximally involutive (i.e., $\overline{\operatorname{Lie}}\left(\Gamma^{\infty}(D)\right)=\mathscr{T}(M)$ ), then the control system will be locally configuration accessible.

We summarise the above discussion with the following result.

4.1 Proposition: Let $\boldsymbol{J}: T M \rightarrow \mathfrak{h}^{*}$ be the momentum map associated with a left action of a Lie group $H$ on $M$. Then:

(i) $D \triangleq \boldsymbol{J}^{-1}(0)$ is a geodesically invariant distribution for the Levi-Civita connection;

(ii) if the control vector fields take their values in $D$, then the trajectories of the control system whose initial velocities are zero are tangent to $D \subset T M$;

(iii) if $\mathscr{Y}=\left\{Y_{1}, \ldots, Y_{m}\right\}$ generates $\Gamma^{\infty}(D)$, then the control system is locally configuration accessible if $D$ is maximally involutive.

\section{Examples}

In this section we present decompositions for a few examples. Some of these examples are presented by Lewis and Murray [1997]. However, in that work the emphasis was on deciding whether the systems were locally configuration accessible. Here we are interested in the case when the systems are not locally configuration accessible and in studying the associated decompositions. In the robotic leg example, we also make connections with Proposition 4.1.

5.1. The robotic leg. We consider a system which consists of a rigid body pinned at its centre of mass and attached is an extendible massless leg with a point mass on its tip. The configuration space is $M=\mathbb{S}^{1} \times \mathbb{S}^{1} \times \mathbb{R}^{+}$. We use the coordinates $(\theta, \psi, r)$ as illustrated in Figure 1. We take $\nabla$ to be the Levi-Civita connection associated with the Riemannian metric

$$
g=J \mathrm{~d} \theta \otimes \mathrm{d} \theta+m r^{2} \mathrm{~d} \psi \otimes \mathrm{d} \psi+m \mathrm{~d} r \otimes \mathrm{d} r
$$

on $M$. Here $J$ is the moment of inertia of the body and $m$ is the value of the point mass on the end of the leg. With this Riemannian metric the system's Lagrangian is $L(v)=\frac{1}{2} g(v, v)$. The non-zero Christoffel symbols are

$$
\Gamma_{\psi r}^{\psi}=\Gamma_{r \psi}^{\psi}=\frac{1}{r}, \quad \Gamma_{\psi \psi}^{r}=-r .
$$

As inputs we choose a force which extends the mass on the end of the leg and a torque which actuates the relative angle between the leg and the body. The corresponding input vector fields are

$$
Y_{1}=\frac{1}{J} \frac{\partial}{\partial \theta}-\frac{1}{m r^{2}} \frac{\partial}{\partial \psi}, \quad Y_{2}=\frac{1}{m} \frac{\partial}{\partial r}
$$

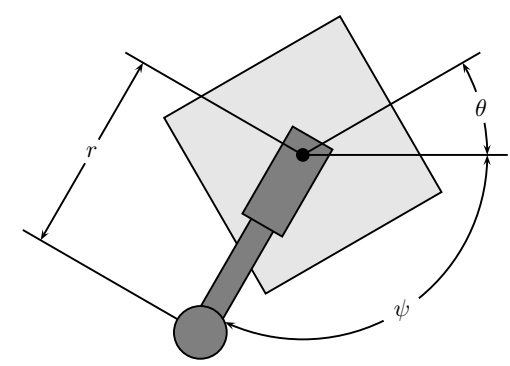

FIgURE 1. The robotic leg

This system admits an action of $H=\mathbb{S}^{1}$ which preserves the metric. The action is given

$$
(\alpha,(\theta, \psi, r)) \mapsto(\theta+\alpha, \psi+\alpha, r) .
$$

The corresponding momentum map is

$$
\boldsymbol{J}\left(\theta, \psi, r, v_{\theta}, v_{\psi}, v_{r}\right)=J v_{\theta}+m r^{2} v_{\psi} .
$$

One may readily verify that $Y_{1}$ and $Y_{2}$ are sections of $D \triangleq \boldsymbol{J}^{-1}(0)$. Thus, by Proposition 4.1(ii), trajectories of the control system whose initial conditions lie on $D$ will remain on $D$. In particular, those trajectories of the control system whose initial velocities are zero will evolve on $D$. Therefore, the system cannot be locally accessible (in $T M$ ). Nevertheless, in [Lewis and Murray 1997] it is shown that the system is locally configuration accessible with both inputs. (In fact, the system is locally configuration controllable with both inputs.) This turns out to be a situation where Proposition 4.1(iii) applies.

Now we consider the case where we just use the input $Y_{2}$. In this case the system is not locally configuration accessible. One may check that $C_{\text {hor }}$ is generated by the vector field $Y_{2}$ itself. The decomposition given by (3.3) takes the form

$$
\begin{aligned}
& \ddot{r}-r \dot{\psi}^{2}=\frac{1}{m} u \\
& \ddot{\theta}=0 \\
& \ddot{\psi}+\frac{2}{r} \dot{r} \dot{\psi}=0 .
\end{aligned}
$$

Observe that if we have zero velocity initial conditions, the values of $\theta$ and $\psi$ are constant so the dynamics are effectively governed by the equation

$$
\ddot{r}=\frac{1}{m} u \text {. }
$$

This is clearly a locally configuration accessible system on each maximal integral manifold of $C_{\text {hor }}$. Physically, this motion is simply one of extending and retracting the mass on the end of the leg while the body itself remains stationary. 


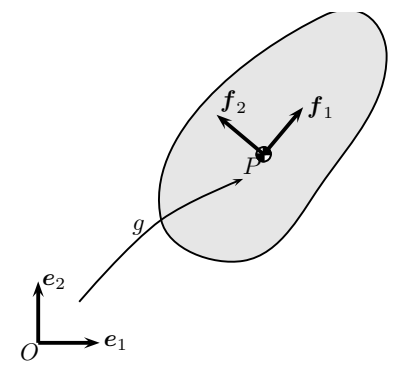

FiguRE 2. The configuration of a planar body as an element of $S E(2)$

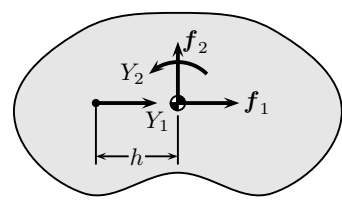

Figure 3. Positions for application of forces on a planar rigid body after simplifying assump-

5.2. The planar rigid body. In this section we study the planar rigid body. The configuration space for the system is the Lie group $S E(2)$. To establish the correspondence between the configuration of the body and $S E(2)$, fix a point $O \in \mathbb{R}^{2}$ and let $\left\{\boldsymbol{e}_{1}=\frac{\partial}{\partial x}, \boldsymbol{e}_{2}=\right.$ $\left.\frac{\partial}{\partial y}\right\}$ be the standard orthonormal frame at that point. Let $\left\{\boldsymbol{f}_{1}, \boldsymbol{f}_{2}\right\}$ be an orthonormal frame attached to the body at its centre of mass. The configuration of the body is determined by the element $g \in S E(2)$ which maps the point $O$ with its frame $\left\{\boldsymbol{e}_{1}, \boldsymbol{e}_{2}\right\}$ to the position, $P$, of the centre of mass of the body with its frame $\left\{\boldsymbol{f}_{1}, \boldsymbol{f}_{2}\right\}$. See Figure 2 . The inputs for this problem consist of a force applied at an arbitrary point and pointing to the centre of mass, and a torque about the centre of mass. Without loss of generality (by redefining our body reference frame $\left\{\boldsymbol{f}_{1}, \boldsymbol{f}_{2}\right\}$ ) we may suppose that the point of application of the force is a distance $h$ along the $\boldsymbol{f}_{1}$ body-axis from the centre of mass. The situation is illustrated in Figure 3.

With this convention fixed, we shall use coordinates $(x, y, \theta)$ for the planar rigid body where $(x, y)$ describe the position of the centre of mass and $\theta$ describes the orientation of the frame $\left\{\boldsymbol{f}_{1}, \boldsymbol{f}_{2}\right\}$ with respect to the frame $\left\{\boldsymbol{e}_{1}, \boldsymbol{e}_{2}\right\}$. In these coordinates, the Riemannian metric for the system is

$$
g=m \mathrm{~d} x \otimes \mathrm{d} x+m \mathrm{~d} y \otimes \mathrm{d} y+J \mathrm{~d} \theta \otimes \mathrm{d} \theta .
$$

Here $m$ is the mass of the body and $J$ is its moment of inertia about the centre of mass The inputs we consider are described by the vector fields

$$
Y_{1}=\frac{\cos \theta}{m} \frac{\partial}{\partial x}+\frac{\sin \theta}{m} \frac{\partial}{\partial y}, \quad Y_{2}=\frac{1}{J} \frac{\partial}{\partial \theta} .
$$

When both of these inputs are applied, the system may be shown to be locally configuration accessible (in fact, controllable). With each of these inputs alone, the system is not locally configuration accessible.

To describe the decomposition (3.3) when we apply the input $Y_{1}$, we need to make a

change of coordinates. To this end we use coordinates

$$
(\xi, \eta, \psi)=(x \cos \theta+y \sin \theta,-x \sin \theta+y \cos \theta, \theta) .
$$

In these coordinates the non-zero Christoffel symbols are

$$
\Gamma_{\eta \psi}^{\xi}=\Gamma_{\psi \eta}^{\xi}=-1, \quad \Gamma_{\psi \psi}^{\xi}=-\xi, \quad \Gamma_{\xi \psi}^{\eta}=\Gamma_{\psi \xi}^{\eta}=1, \quad \Gamma_{\psi \psi}^{\eta}=-\eta .
$$

The distribution $C_{\text {hor }}$ is generated by $\frac{\partial}{\partial \xi}$ in these coordinates. We may now write the system equations as

$$
\begin{aligned}
& \ddot{\xi}-2 \dot{\eta} \dot{\psi}-\xi \dot{\psi}^{2}=\frac{1}{m} u \\
& \ddot{\eta}+2 \dot{\xi} \dot{\psi}-\eta \dot{\psi}^{2}=0 \\
& \ddot{\psi}=0 .
\end{aligned}
$$

Observe that when the initial velocities are zero, the values of $\eta$ and $\psi$ do not change. Thus we essentially have the motion described by the system

$$
\ddot{\xi}=\frac{1}{m} u
$$

which is a locally configuration accessible control system on each maximal integral manifold of $C_{\text {hor }}$. Physically, the motions of this control system are motions of the rigid body along the direction which corresponds to the fixed value of $\theta$. Since the input $Y_{1}$ acts through the centre of mass, the rotational component of the motion is unaffected.

When the system has the input $Y_{2}$ then we may use the coordinates $(x, y, \theta)$ to describe the decomposition (3.3). All Christoffel symbols are zero so the equations for the system are

$$
\begin{aligned}
\ddot{\theta} & =\frac{1}{J} u \\
\ddot{x} & =0 \\
\ddot{y} & =0 .
\end{aligned}
$$

Again we see that the top equation decouples from the last two when the initial velocities are zero. And, as the theory predicts, the top system is locally configuration accessible. The corresponding motion of the system is a rotation about the centre of mass of the body.

5.3. A constrained particle in $\mathbb{R}^{3}$. The example we consider in this section is a mechanical system with constraints. The system has a configuration space of $M=\mathbb{R}^{3}$ and we consider the Riemannian metric

$$
g=m(\mathrm{~d} x \otimes \mathrm{d} x+\mathrm{d} y \otimes \mathrm{d} y+\mathrm{d} z \otimes \mathrm{d} z) .
$$

The system is subject to the velocity constraint

$$
\dot{z}=y \dot{x} .
$$


Lewis [1998] shows that the equations for the constrained motion of this system are equivalent to those geodesics of a certain (non-Levi-Civita) affine connection whose initial velocities satisfy the constraint (5.1). The non-zero Christoffel symbols of this affine connection in the coordinates $(x, y, z)$ are

$$
\Gamma_{x y}^{x}=\frac{2 y}{1+y^{2}}, \quad \Gamma_{z y}^{x}=-\frac{1}{1+y^{2}}, \quad \Gamma_{x y}^{z}=-\frac{1}{1+y^{2}} .
$$

As inputs for this system we consider the single vector field

$$
Y=\frac{1}{m}\left(\frac{\partial}{\partial x}+y \frac{\partial}{\partial z}\right) .
$$

Note that $Y \in \Gamma^{\infty}(D)$ and so this input will not cause the system to violate the constraints. One may verify that the corresponding control system of the form (3.1) is not locally configuration accessible. In fact, the distribution $C_{\text {hor }}$ is generated by the vector field $Y$. This is readily verified by computing $\langle Y: Y\rangle=0$. To render the system in the form (3.3) we must choose coordinates adapted to the foliation associated with $C_{\text {hor }}$. If we choose

$$
(\xi, \eta, \zeta)=(x, y, z-x y)
$$

then one may verify that $C_{\text {hor }}$ is generated by $\frac{\partial}{\partial \xi}$. The non-zero Christoffel symbols in these coordinates are

$$
\begin{gathered}
\Gamma_{\eta \xi}^{\xi}=-\frac{\eta}{1+\eta^{2}}, \quad \Gamma_{\eta \eta}^{\xi}=-\frac{\xi}{1+\eta^{2}}, \quad \Gamma_{\zeta \eta}^{\xi}=-\frac{1}{1+\eta^{2}}, \\
\Gamma_{\xi \eta}^{\zeta}=-\xi, \quad \Gamma_{\eta \xi}^{\zeta}=1-\xi, \quad \Gamma_{\eta \eta}^{\zeta}=\frac{\xi \eta}{1+\eta^{2}}, \quad \Gamma_{\zeta \eta}^{\zeta}=\frac{\eta}{1+\eta^{2}}
\end{gathered}
$$

from which we may derive the governing equations in these coordinates as

$$
\begin{aligned}
& \ddot{\xi}-\frac{\eta}{1+\eta^{2}} \dot{\xi} \dot{\eta}-\frac{\xi}{1+\eta^{2}} \dot{\eta}^{2}-\frac{1}{1+\eta^{2}} \dot{\eta} \dot{\zeta}=\frac{1}{m} u \\
& \ddot{\eta}=0 \\
& \ddot{\zeta}+(1-2 \xi) \dot{\xi} \dot{\eta}+\frac{\xi \eta}{1+\eta^{2}} \dot{\eta}^{2}+\frac{\eta}{1+\eta^{2}} \dot{\eta} \dot{\zeta}=0 .
\end{aligned}
$$

As the general theory predicts, when we have zero initial velocity, the values of $\eta$ and $\zeta$ are not affected by the controls. Thus the system effectively reduces to

$$
\ddot{\xi}=\frac{1}{m} u \text {. }
$$

\section{Discussion}

In this letter we have presented a decomposition of control systems whose drift vector field is the geodesic spray of a general affine connection. Our results illuminate how the geometry of the affine connection interacts with the inputs to specify the reachable sets (Theorem 3.3). It is our belief that this enhanced geometric insight will lead to a better understanding of how one might perform control design for these systems. Furthermore, the possibility of writing the equations of motion for constrained systems in terms of affine connections opens up new possibilities for applying the theory of this paper, and indeed motivates our presentation in terms of general affine connections rather than just LeviCivita connections. We have presented a simple example with constraints to verify that their presence does not affect our conclusions.

\section{References}

Abraham, R. and Marsden, J. E. [1978] Foundations of Mechanics, second edition, Addison Wesley, Reading, MA, ISBN 0-8053-0102-X.

Bloch, A. M. and Crouch, P. E. [1995] Another view of nonholonomic mechanical control systems, in Proceedings of the 34th IEEE Conference on Decision and Control, pages 1066-1071, Institute of Electrical and Electronics Engineers, New Orleans, LA.

Crouch, P. E. [1981] Geometric structures in systems theory, Institution of Electrical Engineers. Proceedings. D. Control Theory and Applications, 128(5), 242-252.

Kobayashi, S. and Nomizu, K. [1963] Foundations of Differential Geometry, Volume I, number 15 in Interscience Tracts in Pure and Applied Mathematics, Interscience Publishers, New York, ISBN 0-470-49647-9.

Lewis, A. D. [1998] Affine connections and distributions with applications to nonholonomic mechanics, Reports on Mathematical Physics, 42(1/2), 135-164.

Lewis, A. D. and Murray, R. M. [1997] Controllability of simple mechanical control systems, SIAM Journal on Control and Optimization, 35(3), 766-790.

Marsden, J. E. and Ratiu, T. S. [1999] Introduction to Mechanics and Symmetry, second edition, number 17 in Texts in Applied Mathematics, Springer-Verlag, New YorkHeidelberg-Berlin, ISBN 0-387-98643-X.

Synge, J. L. [1928] Geodesics in nonholonomic geometry, Mathematische Annalen, 99, 738 